\title{
Count of Unspecified Cells
}

National Cancer Institute

\section{Source}

National Cancer Institute. Count of Unspecified Cells. NCI Thesaurus. Code C112241.

The determination of the amount of unspecified cells, those not otherwise identified, present in a sample. 